\title{
ROBUST STABILITY OF TIME-VARYING DELAY SYSTEMS: THE QUADRATIC SEPARATION APPROACH ${ }^{\dagger}$
}

\author{
Yassine Ariba, Frédéric Gouaisbaut, and Karl Henrik Johansson
}

\begin{abstract}
In this article, we are interested in analysing stability of systems that incorporate time-varying delays in their dynamics. The Lyapunov-Krasovskii approach is definitely the most popular method to address this issue and many results have proposed new functionals and enhanced techniques for deriving less conservative stability conditions. In this present work, we propose an original approach: the quadratic separation. To this end, the delay operator properties are exploited to provide delay range stability conditions. In particular, $L_{2}$-norm of delay-dependent operators are computed so as to reduce the conservatism of the approach. Moreover, the main result is able to assess the stability of non-small delay systems, i.e, it can detect a stability interval for systems that are unstable without any delay. Several examples illustrate the benefit of our methodology.
\end{abstract}

\section{INTRODUCTION}

Time delay system is a subclass of infinite dimensional systems that has been frequently employed since it can easily model commonly arising transport and propagation phenomena. Delays can be encountered in many processes such as biology, chemistry, economics, population dynamics [2] as well as in networks [3]. Unfortunately, delays are the origin of performance and stability degradation, which thus have motivated a lot of work. In the case of constant delay, and unperturbed linear systems, efficient criteria exist based on root loci techniques (see [4] for a recent review). For the case of uncertain linear systems, the problem has been partially solved, either by using Lyapunov functionals $[5,6,7]$ or robustness tools such

Manuscript received July 8, 2008.

Y. Ariba is with the department of Electrical Engineering and Computer Science, Icam, 75 avenue de Grande Bretagne, 31300 Toulouse, France [yassine. ariba@icam. fr].

F. Gouaisbaut is with the LAAS ; CNRS ; Universite de Toulouse, 7 avenue du Colonel Roche, 31077 Toulouse, France [fgouaisbelaas.fr].

K.H. Johansson is with the ACCESS Linnaeus Centre, School of Electrical Engineering, Royal Institute of Technology (KTH), 10044 Stockholm, Sweden. [kallej@kth.se]

$\dagger$ This article is partially based on the conference paper [1] as small gain theorem, integral quadratic constraints [5] and quadratic separation [8]. The proposed conditions are often rather conservative since they produce inner approximations of the stability regions, although recent techniques [8], [9] reduce the conservatism by introducing redundant equations and new decision variables in the optimization problems. Then, these results have been extended to time varying delay systems either using adapted Lyapunov-Krasovskii [9, $10,11,12,13,14,15,16]$ or robustness tools $[17,18$, 19]. These latter methodologies often require, explicitly or implicitly, the delay-free system to be stable, which is a rather important restriction.

This paper aims at providing a novel approach to address time-varying delay system stability. More precisely, we propose criteria based on an extension of the quadratic separation principle [20], [21]. They are then expressed in terms of Linear Matrix Inequalities (LMIs) which may be solved efficiently with SemiDefinite Programming (SDP). In this method, the key idea is how to model the operators that define the system. At first, redundant equations are introduced to construct an augmented model that relates the state vector, its derivative and the delay. Then, a new operator is proposed to refine the modelling of the delay dynamic. At last, using the quadratic separation 
framework with an appropriate modelling, we provide the main result: a delay range stability condition (where the delay $h$ is belonging to a prescribed interval $\left.\left[h_{\min }, h_{\max }\right]\right)$. Differently from most of papers on this topic $[13,22]$, this condition is able to detect pockets of stability even in case of unstable delay-free systems. We emphasize that we do not intend to present an additional less conservative criterion that outperforms all existing results for non small delays but rather an original methodology to cope with systems which are unstable for sufficiently small delays.

The outline of the paper is as follows. In Section II, some preliminaries are presented and we state our quadratic separation theorem as well as a set of useful operators. In section III, this latter prior result is exploited to derive a stability condition for timevarying delay systems. Then, an additional operator is inserted for the conservatism reduction. At last, numerical examples that show the effectiveness of the proposed criterion are provided in section IV. Section $\mathrm{V}$ concludes the paper.

Notations: Throughout the paper, the following notations are used. The set $L_{2}^{n}$ consists of all measurable functions $f: \mathbb{R}^{+} \rightarrow \mathbb{C}^{n}$ such $\|f\|_{L_{2}}=$ $\left(\int_{0}^{\infty}\left(f^{*}(t) f(t)\right)\right)^{1 / 2} d t<\infty$. When context allows it, the superscript $n$ of the dimension will be omitted. The set $L_{2 e}^{n}$ denotes the extended set of $L_{2}^{n}$ which consists of the functions whose time truncation lies in $L_{2}^{n}$. For two symmetric matrices, $A$ and $B, A>(\geq) B$ means that $A-B$ is (semi-)positive definite. $A^{T}$ denotes the transpose of $A .1_{\mathrm{n}}$ and $0_{\mathrm{m} \times \mathrm{n}}$ denote, respectively, the identity matrix of size $n$ and null matrix of size $m \times$ $n$. If the context allows it, the dimensions of these matrices are omitted. $\operatorname{diag}\left(A_{1}, \ldots, A_{k}\right)$ stands for the block diagonal matrix with $A_{1}, \ldots, A_{k}$ on the diagonal. Introduce as well the truncation operator $\mathbb{P}_{T}$ such that:

$$
\mathbb{P}_{T}(f)=f_{T}=\left\{\begin{array}{cc}
f(t), & t \leq T \\
0, & t>T
\end{array}\right.
$$

\section{PRELIMINARIES}

\subsection{Problem statement}

Consider the following time-varying delay system:

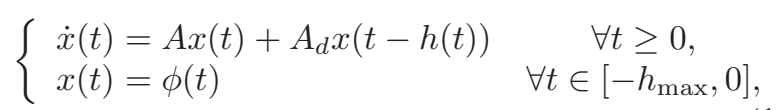

where $x(t) \in \mathbb{R}^{n}$ is the state vector, $\phi$ is the initial condition and $A, A_{d} \in \mathbb{R}^{n \times n}$ are constant matrices. The delay $h$ is time varying with

$$
h(t) \in\left[h_{\min }, h_{\max }\right] \text { and }|\dot{h}(t)| \leq d,
$$

where $h_{\min }, h_{\max }$ and $d$ are given positive constants. In this work, we aim at assessing the stability of system (1) via the quadratic separation principle originally developed for robust control in [23]. We will show that various criteria, related to the available informations on the delay, can be derived choosing appropriately a set of operators.

\subsection{Stability analysis via quadratic separation}

The quadratic separation provides a fruitful framework to address stability of non-linear and uncertain systems [20], [21]. Recent studies [8] have shown that such a framework reduces significantly the conservatism of the stability analysis of time-delay systems with constant delay. In this paper we extend this method to time varying delay systems, which involves the development of results for a new set of operators. Consider the interconnection in Figure 1 where $\mathcal{E}$ and $\mathcal{A}$ are two, real valued, possibly non-square, matrices and $\nabla$ is a linear operator from $L_{2 e}$ to $L_{2 e}$. For simplicity, we assume that $\mathcal{E}$ is full column rank. Assuming wellposedness, we are interested in looking for conditions that ensure stability of the interconnection.

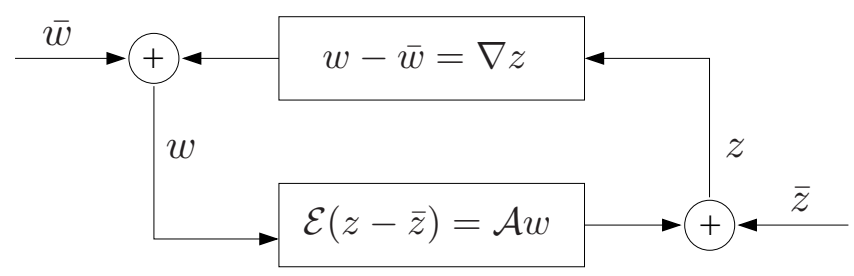

Fig. 1. Feedback system.

Theorem 1 The interconnected system of Figure 1 is stable if there exists a symmetric matrix $\Theta=\Theta^{T}$ satisfying both conditions

$$
\left[\begin{array}{ll}
\mathcal{E} & -\mathcal{A}
\end{array}\right]^{\perp^{T}} \Theta\left[\begin{array}{ll}
\mathcal{E} & -\mathcal{A}
\end{array}\right]^{\perp}>0
$$

$$
\forall u \in L_{2 e}, \forall T>0,\left\langle\left[\begin{array}{c}
1 \\
\mathbb{P}_{T} \nabla
\end{array}\right] u_{T}, \Theta\left[\begin{array}{c}
1 \\
\mathbb{P}_{T} \nabla
\end{array}\right] u_{T}\right\rangle \leq 0
$$


PROOF : Inspired from [20], the proof is detailed in [18].

This result includes two conditions: a matrix inequality (3) related to the lower block of the feedback system and an inner product (4) that states an Integral Quadratic Constraint (IQC) on the upper block. It will be used throughout the paper to prove stability of systems under consideration.

\subsection{Some suitable operators}

It is required to define appropriate operators to model the time-delay system (1) as the feedback system in Figure 1. Clearly, two operators are essential, reflecting the dynamics and the delay: the integral operator

$$
\begin{aligned}
\mathcal{I}: L_{2 e} & \rightarrow L_{2 e}, \\
x(t) & \rightarrow \int_{0}^{t} x(\theta) d \theta,
\end{aligned}
$$

and the delay operator (or shift operator)

$$
\begin{aligned}
\mathcal{D}: & L_{2 e} \rightarrow L_{2 e}, \\
& x(t) \rightarrow x(t-h),
\end{aligned}
$$

The next step is to characterize the two operators by the use of IQCs introduced in the following two lemmas, which are used to derive stability criteria for time-delay systems in the next section.

Lemma 1 An IQC for the operator $\mathcal{I}$ is given by the following inequality: $\forall x \in L_{2 e}^{n}$ and $\forall P>0$,

$$
\left\langle\left[\begin{array}{c}
1_{\mathrm{n}} \\
\mathbb{P}_{T} \mathcal{I} 1_{\mathrm{n}}
\end{array}\right] x_{T},\left[\begin{array}{cc}
0 & -P \\
-P & 0
\end{array}\right]\left[\begin{array}{c}
1_{\mathrm{n}} \\
\mathbb{P}_{T}^{\mathcal{I} 1_{\mathrm{n}}}
\end{array}\right] x_{T}\right\rangle \leq 0 .
$$

Proof : Simple calculus shows that $\forall T>0, \forall x \in L_{2 e}^{n}$,

$$
\begin{aligned}
& \left\langle\left[\begin{array}{c}
1_{\mathrm{n}} \\
\mathcal{I} 1_{\mathrm{n}}
\end{array}\right] x,\left[\begin{array}{cc}
0 & -P \\
-P & 0
\end{array}\right]\left[\begin{array}{c}
1_{\mathrm{n}} \\
\mathcal{I} 1_{\mathrm{n}}
\end{array}\right] x\right\rangle \\
& =-2 \int_{0}^{T} x(t)^{T} P \int_{0}^{t} x(s) d s d t \\
& =-2 \int_{0}^{T} \frac{d}{d t}(\mathcal{I} x)^{T} P(\mathcal{I} x) d t \\
& =-\left(\int_{0}^{T} x(s) d s\right)^{T} P\left(\int_{0}^{T} x(s) d s\right) \leq 0
\end{aligned}
$$

Lemma 2 An IQC for the operator $\mathcal{D}$ is given by the following inequality: $\forall T>0, \forall x \in L_{2 e}^{n}$ and $\forall Q>0$,

$\left\langle\left[\begin{array}{c}1_{\mathrm{n}} \\ \mathbb{P}_{T} \mathcal{D} 1_{\mathrm{n}}\end{array}\right] x_{T},\left[\begin{array}{cc}-Q & 0 \\ 0 & Q(1-\dot{h})\end{array}\right]\left[\begin{array}{c}1_{\mathrm{n}} \\ \mathbb{P}_{T} \mathcal{D} 1_{\mathrm{n}}\end{array}\right] x_{T}\right\rangle \leq 0$.

Proof : We get that $\forall T>0, \forall x \in L_{2 e}^{n}$,

$$
\begin{aligned}
& \left\langle\left[\begin{array}{c}
1_{\mathrm{n}} \\
\mathcal{D} 1_{\mathrm{n}}
\end{array}\right] x,\left[\begin{array}{cc}
-Q & 0 \\
0 & Q(1-\dot{h})
\end{array}\right]\left[\begin{array}{c}
1_{\mathrm{n}} \\
\mathcal{D} 1_{\mathrm{n}}
\end{array}\right] x\right\rangle \\
& =-\int_{0}^{+\infty} x^{T}(u) Q x(u) d u+\int_{0}^{\infty} x_{d}^{T}(t) Q x_{d}(t)(1-\dot{h}(t)) d t \\
& =-\int_{0}^{+T} x^{T}(t) Q x(t) d t+\int_{-h(0)}^{T-h(T)} x^{T}(u) Q x(u) d u \\
& =-\int_{T-h(T)}^{T} x(u)^{T} Q x(u) d u \leq 0
\end{aligned}
$$

where $x_{d}(t)=x(t-h(t))$.

Since the IQC for the delay operator $\mathcal{D}$ do not depend on $h$, it is clear that it will induce some conservatism. As an example, in the constant delay case, the IQC defined by Lemma 2 is equivalent to that the delay operator is replaced by a norm-bounded uncertainty. The phase of $e^{-h s}$ is not taken into account. This can be approached by the operator $\delta_{1}(s)=\frac{1-e^{-h s}}{s}$, [19]. It can be embedded as a norm bounded uncertainty:

$$
\sup _{\omega}\left\|\frac{1-e^{-j \omega h}}{j \omega}\right\| \leq h_{\max } .
$$

The operator can also be interpreted in the first-order Taylor remainder of the exponential function $e^{-h s}$ :

$$
e^{-h s}=1-h s \delta_{1}(s) .
$$

Following the same idea, we formulate now the timevarying counterpart by considering a new operator $\mathcal{F}$ defined as follows:

$$
\begin{aligned}
\mathcal{F}: \quad L_{2 e} & \rightarrow L_{2 e}, \\
x(t) & \rightarrow \int_{t-h(t)}^{t} x(s) d s .
\end{aligned}
$$

Its characterization through an IQC can be derived as follows:

Lemma 3 An IQC for the operator $\mathcal{F}=(1-\mathcal{D}) \circ \mathcal{I}$ is given by the following inequality: $\forall x \in L_{2 e}^{n}$,

$\left\langle\left[\begin{array}{c}1_{\mathrm{n}} \\ \mathbb{P}_{T} \mathcal{F} 1_{\mathrm{n}}\end{array}\right] x_{T},\left[\begin{array}{cc}-h_{\max }^{2} R & 0 \\ 0 & R\end{array}\right]\left[\begin{array}{c}1_{\mathrm{n}} \\ \mathbb{P}_{T} \mathcal{F} 1_{\mathrm{n}}\end{array}\right] x_{T}\right\rangle \leq 0$

where $h_{\max }$ is the upperbound on the delay $h(t)$ and $R$ is some positive definite matrix. 
ProOF : See [19] or [18].

The operator $\mathcal{F}=(1-\mathcal{D}) \circ \mathcal{I}$ can be slightly transformed as $\overline{\mathcal{F}}=\frac{1}{h(t)} \mathcal{F}$. The corresponding integral constraint is then expressed as follows.

Lemma 4 An IQC for the operator $\overline{\mathcal{F}}$ is given by the following inequality: $\forall T>0, \forall x \in L_{2 e}^{n}, \forall R>0$, inequality,

$$
\begin{gathered}
\|\tilde{\mathcal{H}} x\|^{2} \leq\left(\int_{t-h(t)}^{t} \int_{s}^{t} d \theta d s\right)\left(\int_{t-h(t)}^{t} \int_{s}^{t}\|x(\theta)\|^{2} d \theta d s\right), \\
\frac{\|\tilde{\mathcal{H}} x\|^{2}}{h^{2}(t) / 2} \leq \int_{t-h(t)}^{t} \int_{s}^{t}\|x(\theta)\|^{2} d \theta d s, \\
\int_{0}^{\infty} \frac{2}{h^{2}(t)}\|\tilde{\mathcal{H}} x\|^{2} d t \leq \int_{0}^{\infty} \int_{-h_{\max }}^{0} \int_{0}^{0}\left\|x_{t}(\theta)\right\|^{2} d \theta d s d t \\
\int_{0}^{\infty} \frac{2}{h^{2}(t)}\|\tilde{\mathcal{H}} x\|^{2} d t \leq \frac{h_{\max }^{2}}{2} \int_{0}^{\infty}\|x(t)\|^{2} d t .
\end{gathered}
$$

ProOF : Omitted.

An interesting contribution of this work is then the introduction of a novel operator that improves the modelling of the delay dynamic. This operator is related to the Taylor remainder of order two:

$\mathcal{H}=\frac{\mathcal{I}^{2}-\mathcal{D} \mathcal{I}^{2}-h(t) \mathcal{I}}{h(t)}: x(t) \rightarrow \frac{1}{h(t)} \int_{t-h(t)}^{t} \int_{s}^{t} x(\theta) d \theta d s$

The following lemma gives a parameterized constraint on $\mathcal{H}$.

Lemma 5 An IQC for the operator $\mathcal{H}$ is given by the following inequality: $\forall T>0, \forall x \in L_{2 e}^{n}, \forall S>0$,

$\left\langle\left[\begin{array}{c}1_{\mathrm{n}} \\ \mathbb{P}_{T} \mathcal{H} 1_{\mathrm{n}}\end{array}\right] x_{T},\left[\begin{array}{cc}-\frac{h_{\max }^{2}}{2} S & 0 \\ 0 & 2 S\end{array}\right]\left[\begin{array}{c}1_{\mathrm{n}} \\ \mathbb{P}_{T} \mathcal{H} 1_{\mathrm{n}}\end{array}\right] x_{T}\right\rangle \leq 0$

which concludes the proof.

This result is a key result for the main theorem because it allows to build a stability condition that does not require the system to be stable for small delays as we will see in section IV.

\section{MAIN RESULTS}

We present in this section the main results of the article which is based on the quadratic separation framework already used for constant delay systems. This approach allows us to establish the main theorem for the robust delay range stability analysis.

\subsection{Methodology}

PROOF : Note that

To illustrate the proposed methodology, let us reformulate the system (1) as the feedback in Figure 1. As a first modelling, the system (1) can be described as $\|\mathcal{H} x\|^{2}=\frac{1}{h^{2}(t)}\left(\int_{t-h(t)}^{t} \int_{s}^{t} x(\theta) d \theta d s\right)^{T}\left(\int_{t-h(t)}^{t} \int_{s}^{t} x(\theta) d \theta d s\right)^{t}$

Using Cauchy-Schwartz inequality and setting $\tilde{\mathcal{H}}=$ the feedback

$$
\underbrace{\left[\begin{array}{c}
x(t) \\
x(t-h(t)) \\
x(t)-x(t-h(t))
\end{array}\right]}_{w(t)}=\underbrace{\left[\begin{array}{ccc}
\mathcal{I} 1_{\mathrm{n}} & 0 & 0 \\
0 & \mathcal{D} 1_{\mathrm{n}} & 0 \\
0 & 0 & \mathcal{F} 1_{\mathrm{n}}
\end{array}\right]}_{\nabla} \underbrace{\left[\begin{array}{c}
\dot{x}(t) \\
x(t) \\
\dot{x}(t)
\end{array}\right]}_{z(t)},
$$


with

$$
\underbrace{\left[\begin{array}{ccc}
1 & 0 & 0 \\
0 & 1 & 0 \\
-1 & 0 & 1 \\
0 & 0 & 0
\end{array}\right]}_{\mathcal{E}} z(t)=\underbrace{\left[\begin{array}{ccc}
A & A_{d} & 0 \\
1 & 0 & 0 \\
0 & 0 & 0 \\
1 & -1 & -1
\end{array}\right]}_{\mathcal{A}} w(t) .
$$

Then, according to Theorem 1, we have to find a separator $\Theta$ that fulfills both inequalities (3)-(4). Combining the three constraints related to the different operators (stated by the lemmas in Section 2.3), a global (conservative) constraint on $\nabla$ is deduced. Hence, the matrix

$\Theta=\left[\begin{array}{ccc|ccc}0 & 0 & 0 & -P & 0 & 0 \\ 0 & -Q & 0 & 0 & 0 & 0 \\ 0 & 0 & -h_{\max }^{2} R & 0 & 0 & 0 \\ \hline-P & 0 & 0 & 0 & 0 & 0 \\ 0 & 0 & 0 & 0 & Q(1-\dot{h}(t)) & 0 \\ 0 & 0 & 0 & 0 & 0 & R\end{array}\right]$

where $P, Q$ and $R$ are $n \times n$ positive definite matrices, satisfies the inequality (4). The interconnected system (10)-(11) (and therefore system (1)) is thus stable if the matrix inequality (3), with $\mathcal{E}, \mathcal{A}$ and $\Theta$ defined as (11) and (12), holds. Because of the occurrences of $h_{\max }$ and $\dot{h}(t)$ in the criterion, it is refered to as delay and rate dependent. Setting $\dot{h}(t)=d$ in the separator, the condition becomes a single LMI that can be easily solved via SDP.

Remark 1 It has been shown in [18] that the above criterion, based on the three operators, provides the same results in terms of conservativeness as several classical results of the literature [6, 24]. Indeed, such a particular choice of operators and separator amounts to choosing a Lyapunov-Krasovskii functional candidate of the form:

$$
\begin{aligned}
V\left(x_{t}\right)=x_{t}^{T}(0) P x_{t}(0) & +\int_{-h(t)}^{0} x_{t}^{T}(\theta) Q x_{t}(\theta) d \theta \\
& +\int_{t-h_{m}}^{0} \int_{\theta}^{0} \dot{x}_{t}^{T}(s) R \dot{x}_{t}(s) d s d \theta .
\end{aligned}
$$

Further discussions on the quadratic separation method and the Lyapunov-Krasovskii counterpart for the constant delay case can be found in [8]. Other authors have emphasized the links between the Lyapunov method and the robust analysis in general, e.g., [11, 23, 25].
Remark 2 A simpler criterion can be derived by removing $\mathcal{F}$ from $\nabla$. In that case, the stability condition is independent of the delay because no information on the size of $h(t)$ (for instance, $h_{\max }$ ) appears in the matrices $\mathcal{E}, \mathcal{A}$ and $\Theta$. However, a bound on $\dot{h}$ is still required.

Remark 3 Because the inequality in the Lemma 2 imposes a constraint on the delay variation $\dot{h}(t)$, a rate independent condition can be obtained if the system (1) is represented only through the first and the third operators of (10).

In the next sections, we investigate new operators for the delayed dynamics. The objective is to reduce the conservatism of the stability analysis by taking into account some further informations on the delay. Throughout the paper we will apply the following procedure:

(a) Rewrite the delay system (1) as an interconnected feedback.

(b) Embed the integrator, the delay and other auxiliary operators into the matrix $\nabla$.

(c) Construct IQCs for $\nabla$.

(d) Establish the LMIs of Theorem 1 and compute the separator $\Theta$.

\subsection{Model extension}

By extending the dynamics of the time-delay system, it is possible to achieve less conservative results, see $[26,8,27]$. An augmented state is composed of the original state vector and its derivative. By defining relationship between augmented state $\dot{x}, \ddot{x}$, the delay $h$ and its derivative $\dot{h}$, an enhanced stability condition is provided. Differentiating the system (1), we get:

$$
\ddot{x}(t)=A \dot{x}(t)+(1-\dot{h}(t)) A_{d} \dot{x}(t-h(t)) .
$$

Consider

$$
\left\{\begin{array}{l}
\dot{x}(t)=A x(t)+A_{d} x(t-h(t)) \\
\ddot{x}(t)=A \dot{x}(t)+(1-\dot{h}(t)) A_{d} \dot{x}(t-h(t)) .
\end{array}\right.
$$

Introduce the augmented state

$$
\varsigma(t)=\left[\begin{array}{l}
\dot{x}(t) \\
x(t)
\end{array}\right]
$$


so by specifying the relationship between the two components of $\varsigma(t)$ with the equality $\left[\begin{array}{ll}0 & 1\end{array}\right] \dot{\zeta}(\mathrm{t})=$ $\left[\begin{array}{ll}1 & 0\end{array}\right] \varsigma(\mathrm{t})$, we have the descriptor system

$$
E \dot{\varsigma}(t)=\bar{A} \varsigma(t)+\bar{A}_{d} \varsigma(t-h(t)),
$$

where

$$
\begin{gathered}
E=\left[\begin{array}{ll}
1 & 0 \\
0 & 1 \\
1 & 0
\end{array}\right], \bar{A}=\left[\begin{array}{cc}
A & 0 \\
0 & A \\
0 & 1
\end{array}\right], \\
\bar{A}_{d}=\left[\begin{array}{cc}
A_{d} & 0 \\
0 & (1-\dot{h}(t)) A_{d} \\
0 & 0
\end{array}\right] .
\end{gathered}
$$

\subsection{Delay range stability condition}

We now model the augmented time-varying delay system (15) through the new set of operators:

$$
\underbrace{\left[\begin{array}{c}
\varsigma(t) \\
\varsigma_{d}(t) \\
w_{1}(t) \\
w_{2}(t)
\end{array}\right]}_{w(t)}=\underbrace{\left[\begin{array}{cccc}
\mathcal{I} 1_{2 \mathrm{n}} & 0 & 0 & 0 \\
0 & \mathcal{D} 1_{2 \mathrm{n}} & 0 & 0 \\
0 & 0 & \overline{\mathcal{F}} 1_{2 \mathrm{n}} & 0 \\
0 & 0 & 0 & \mathcal{H} 1_{\mathrm{n}}
\end{array}\right]}_{\nabla} \underbrace{\left[\begin{array}{c}
\dot{\zeta}(t) \\
\varsigma(t) \\
\dot{\zeta}(t) \\
\ddot{x}(t)
\end{array}\right]}_{z(t)}
$$

with

$$
\begin{aligned}
\varsigma_{d}(t) & =\varsigma(t-h(t)), \\
w_{1}(t) & =\frac{\varsigma(t)-\varsigma(t-h(t))}{h(t)}, \\
w_{2}(t) & =\dot{x}(t)-\frac{x(t)-x(t-h(t))}{h(t)}=E_{1} \varsigma(t)-E_{2} w_{1}(t)
\end{aligned}
$$

and $E_{1}=\left[\begin{array}{ll}1 & 0\end{array}\right]$ and $E_{2}=\left[\begin{array}{ll}0 & 1\end{array}\right]$. Then, according to the Lemmas (1)-(5), the separator

$$
\begin{gathered}
\Theta=\left[\begin{array}{c|c}
\Theta_{11} & \Theta_{12} \\
\hline * & \Theta_{22}
\end{array}\right], \\
\Theta_{11}=\operatorname{diag}\left(0_{2 \mathrm{n}},-Q,-h_{\max } R,-\frac{h_{\max }^{2}}{2} S\right), \\
\Theta_{12}=\operatorname{diag}\left(-P, 0_{5 \mathrm{n}}\right), \\
\Theta_{22}=\operatorname{diag}\left(0_{2 \mathrm{n}},(1-\dot{h}(t)) Q, h(t) R, 2 S\right),
\end{gathered}
$$

with some positive definite matrices $P, Q, R \in \mathbb{R}^{2 n \times 2 n}$ and $S \in \mathbb{R}^{n \times n}$, fulfils the requirement (4) of Theorem 1. Consequently, the stability of (15) (and thus (1)) holds if

$$
\xi^{T}(t) \Theta(h(t), \dot{h}(t)) \xi(t)>0
$$

such that $\left[\begin{array}{ll}\mathcal{E} & -\mathcal{A}\end{array}\right] \xi(t)=0$ with $\xi=\left[\begin{array}{c}z(t) \\ w(t)\end{array}\right]$. This condition is equivalent to (3) of Theorem 1. Condition (18) can be rewritten as another equivalent condition

$$
\psi^{T}(t) N^{T}(\dot{h}(t)) \Theta(\dot{h}(t)) N(\dot{h}(t)) \psi(t)>0,
$$

where $\psi=\left[\begin{array}{c}x(t) \\ \varsigma(t-h(t)) \\ w_{1}(t) \\ w_{2}(t)\end{array}\right]$, such that $S(h(t)) \psi(t)=0$ with

$$
S=\left[\begin{array}{cccccc}
A & -1 & A_{d} & -1 h(t) & 0 & 0 \\
1 & 0 & -1 & 0 & -1 h(t) & 0 \\
A & 0 & A_{d} & 0 & -1 & -1
\end{array}\right]
$$

and

$$
N=\left[\begin{array}{ccc|c}
A A & A_{d}(1-\dot{h}) & A A_{d} & \\
A & 0 & A_{d} & \\
A & 0 & A_{d} & \\
1 & 0 & 0 & 0_{8 \mathrm{n} \times 3 \mathrm{n}} \\
A A & A_{d}(1-\dot{h}) & A A_{d} & \\
A & 0 & A_{d} & \\
A A & A_{d}(1-\dot{h}) & A A_{d} & \\
A & 0 & A_{d} & \\
\hline \multicolumn{5}{c}{1_{6 \mathrm{n}}}
\end{array}\right]
$$

Applying Finsler's lemma, we note that condition (19) is equivalent to

$$
N^{T}(\dot{h}(t)) \Theta N(\dot{h}(t))+X S(h(t))+S^{T}(h(t)) X^{T}>0 .
$$

It is easy to show that $N^{T}(\dot{h}(t)) \Theta N(\dot{h}(t))$ is affine, and thus convex, in $h$ and $\dot{h}$. So condition (22) has to be assessed only at the four vertices of the polytop generated by the intervals of $h(t)$ and $\dot{h}(t)$. We are now in a position to state our main result.

Theorem 2 For given positive scalars $d, h_{\min }$ and $h_{\max }$, if there exist positive definite matrices $P, Q$, $R \in \mathbb{R}^{2 n \times 2 n}$, a positive definite matrix $S \in \mathbb{R}^{n \times n}$ and a matrix $X \in \mathbb{R}^{6 n \times 3 n}$, then the system (1) with a time-varying delay constrained by (2) is asymptotically stable if the LMI (22) holds for $\dot{h}(t) \in\{-d, d\}$ and $h(t) \in\left\{h_{\min }, h_{\max }\right\}$.

Remark 4 Most of the papers in the literature provide the so-called delay dependent stability condition using the Lyapunov-Krasovskii method (see for example [6, 24, 28, 29]). Basically, a stable delay-free system 
is considered and the maximal value of the delay that preserves the stability is looked for. Recently, some papers have studied the problem of finding the largest delay interval $\left[h_{\min }, h_{\max }\right]$ for which the delay system is stable. In that case, the Lyapunov-Krasovskii functionnal depends explicitly on the delay $h(t)$, but also on the lower and upper bound [30, 13, 14, 22]. In these papers, they explore tightly the relations between $x\left(t-h_{\min }\right)$ and $x\left(t-h_{\max }\right)$ through the use of wellfitted Lyapunov-Krasovskii functionals. Nevertheless, their results are restricted to the case of a stable delay free system, i.e. a stable matrix $A+A_{d}$. We address in this paper the tricky case of the delay range condition where the delay belongs to an interval $(h(t) \in$ $\left.\left[h_{\min }, h_{\max }\right]\right)$ and the system may be unstable for small delays.

\subsection{Robust stability}

Quadratic separation provides a suitable framework for stability analysis of uncertain delay systems:

$$
\dot{x}(t)=A(\Delta) x(t)+A_{d}(\Delta) x(t-h(t))
$$

where

$$
\left[\begin{array}{ll}
A(\Delta) & A_{d}(\Delta)
\end{array}\right]=\left[\begin{array}{ll}
A & A_{d}
\end{array}\right]+B \Delta\left[\begin{array}{ll}
C & C_{d}
\end{array}\right] .
$$

The second term of the right hand side describes the uncertainty characterizing system (23). The uncertain time-varying matrix $\Delta(t)$ satisfies

$$
\Delta^{T}(t) \Delta(t) \leq 1, \forall \mathrm{t} \geq 0, \forall \Delta \in \Omega,
$$

and models non-linear and neglected dynamics as well as parametric uncertainties. The matrices $B, C$ and $C_{d}$ are constant and of appropriate dimensions. According to the set of admissible uncertainties and (24), we have to find a separator $U$ such that

$$
\langle\left[\begin{array}{c}
1 \\
\Delta
\end{array}\right] x, \underbrace{\left[\begin{array}{cc}
U_{1} & U_{2} \\
U_{2}^{*} & U_{3}
\end{array}\right]}_{U}\left[\begin{array}{c}
1 \\
\Delta
\end{array}\right] x\rangle<0, \forall \Delta \in \Omega .
$$

For instance, assume $\Omega$ is a set of diagonal real-valued matrices with bounded uncertainties:

$$
\Omega=\left\{\Delta=\operatorname{diag}\left(\delta_{1}, \ldots, \delta_{N}\right)|| \delta_{i} \mid \leq \bar{\delta}_{i}\right\} .
$$

Then, inequality (25) holds with

$$
U=\operatorname{diag}\left(-\bar{\delta}_{1}^{2} u_{1}, \ldots,-\bar{\delta}_{N}^{2} u_{N}, u_{1}, \ldots, u_{N}\right)
$$

where $u_{i}, i=1, \ldots, N$, are scalar decision variables. We propose to analyze the robust stability of system (23) with the following theorem.
Theorem 3 For given positive scalars $h_{\max }$ and $d$, if there exists positive definite matrices $P, Q, R \in \mathbb{R}^{n \times n}$ and matrices $U_{1}, U_{2}, U_{3}$ such that (25) holds, then system (23) with a time-varying delay constrained by (2) is asymptotically stable for any uncertainty $\Delta \in \Omega$ if the LMI condition (3) holds with $\Theta, \mathcal{E}$ and $\mathcal{A}$ defined as follows:

$$
\begin{aligned}
& \Theta=\left[\begin{array}{ll}
\Theta_{11} & \Theta_{12} \\
\Theta_{12}^{*} & \Theta_{22}
\end{array}\right], \begin{array}{l}
\Theta_{11}=\operatorname{diag}\left(0_{\mathrm{n}},-Q,-h_{\max }^{2} R, U_{1}\right), \\
\Theta_{12}=\operatorname{diag}\left(-P, 0_{2 \mathrm{n}}, U_{2}\right), \\
\Theta_{22}=\operatorname{diag}\left(0_{\mathrm{n}},(1-d) Q, R, U_{3}\right),
\end{array} \\
& \mathcal{E}=\left[\begin{array}{cccc}
1_{\mathrm{n}} & 0 & 0 & 0 \\
0 & 1_{\mathrm{n}} & 0 & 0 \\
-1_{\mathrm{n}} & 0 & 1_{\mathrm{n}} & 0 \\
0 & 0 & 0 & 1_{\mathrm{n}} \\
0 & 0 & 0 & 0
\end{array}\right] \\
& \mathcal{A}=\left[\begin{array}{cccc}
A & A_{d} & 0 & B \\
1_{\mathrm{n}} & 0 & 0 & 0 \\
0 & 0 & 0 & 0 \\
C & C_{d} & 0 & 0 \\
1_{\mathrm{n}} & -1 & -1 & 0
\end{array}\right] \text {. }
\end{aligned}
$$

PROOF : First, introducing the exogenous signals

$$
w_{\Delta}=\Delta z_{\Delta}, \text { with } z_{\Delta}=C x(t)+C_{d} x(t-h(t)),
$$

we rewritte system (23) as the interconnection of

$$
\underbrace{\left[\begin{array}{c}
x(t) \\
x(t-h(t)) \\
x(t)-x(t-h(t)) \\
w_{\Delta}(t)
\end{array}\right]}_{w(t)}=\nabla \underbrace{\left[\begin{array}{c}
\dot{x}(t) \\
x(t) \\
\dot{x}(t) \\
z_{\Delta}(t)
\end{array}\right]}_{z(t)}
$$

with $\nabla=\left(\mathcal{I} 1_{\mathrm{n}}, \mathcal{D} 1_{\mathrm{n}}, \mathcal{F} 1_{\mathrm{n}}, \Delta\right)$ and

$$
\mathcal{E} z(t)=\mathcal{A} w(t)
$$

$\mathcal{E}$ and $\mathcal{A}$ are defined in (27). Combining every IQC related to each operators defined by lemmas and the struture of the uncertainty leading to (25), a separator of the form of (26) fulfills the requirement (4). Finally, condition (3) provides the robust (with respect to the uncertain set $\Omega$ ) stability criterion.

For the sake of simplicity, Theorem 3 is given only with the two operators $\mathcal{D}$ and $\mathcal{F}$. In the case of time invariant uncertainties, it is easy to extend to the third operator $\mathcal{H}$. If $\Delta$ is time-dependent, the model extension (Subsection 3.2) is however more tricky to apply and a good knowledge of the uncertainty is required. 


\section{NUMERICAL EXAMPLES}

We illustrate the developed theory through three examples.

\subsection{First example: delay dependent case}

Consider

$\dot{x}(t)=\left[\begin{array}{cc}-2 & 0 \\ 0 & -0.9\end{array}\right] x(t)+\left[\begin{array}{cc}-1 & 0 \\ -1 & -1\end{array}\right] x(t-h(t))$.

First, let us remark that the delay-free case is stable. Next, the maximal allowable delay, $h_{\max }$, is computed. To demonstrate the effectiveness of our approach, results are compared to the literature. All papers, except $[17,19,31]$, use Lyapunov theory in order to derive stability criteria. In [17], [19], the stability problem is solved in an IQC framework. The results are shown in Table 1.

In [19] and [31], the delay is modeled as an uncertain parameter and appropriate weighting filters are used to bound it. Their methodologies provide very good results, however, they are restricted to time-delay system that are stable without delay. Although Theorem 2 does not provide the best condition, it provides conservatism reduction compared to many conditions from the literature. Besides, Theorem 2 addresses the stability of systems with interval delays, which may be unstable for small delays (or without delays).

\subsection{Second example: delay range case}

Consider $\ddot{y}(t)-0.1 \dot{y}(t)+2 y(t)=u(t)$, with a static delayed output feedback $u(t)=k y(t-h(t))$. Choosing $k=1$, we get:

$$
\dot{x}(t)=\left[\begin{array}{cc}
0 & 1 \\
-2 & 0.1
\end{array}\right] x(t)+\left[\begin{array}{ll}
0 & 0 \\
1 & 0
\end{array}\right] x(t-h(t)) .
$$

In order to assess the interval of the delay such that system (30) is stable, Theorem 2 is applied with given $h_{\min }$ and $h_{\max }$. Then, a sliding window principle is performed to stretch the bounds. The results are presented in Table 2.

Theorem 2 allows us to assess a conservative region of stability w.r.t. $k$ and $h(t)$ (for $d=1$ ). It provides a set of values of $k$ that ensures a stabilizing delayed output feedback for $\ddot{y}(t)-0.1 \dot{y}(t)+2 y(t)=$ $u(t)$ as shown in Figure 2.
Table 2. Interval of stabilizing delays for system (30)

\begin{tabular}{lll}
\hline $\mathrm{d}$ & $h_{\min }$ & $h_{\max }$ \\
\hline 0 & 0.102 & 1.424 \\
0.1 & 0.102 & 1.424 \\
0.2 & 0.103 & 1.423 \\
0.5 & 0.104 & 1.421 \\
0.8 & 0.105 & 1.419 \\
1.0 & 0.105 & 1.418 \\
0 (analytical) & 0.10016826 & 1.7178 \\
\hline
\end{tabular}

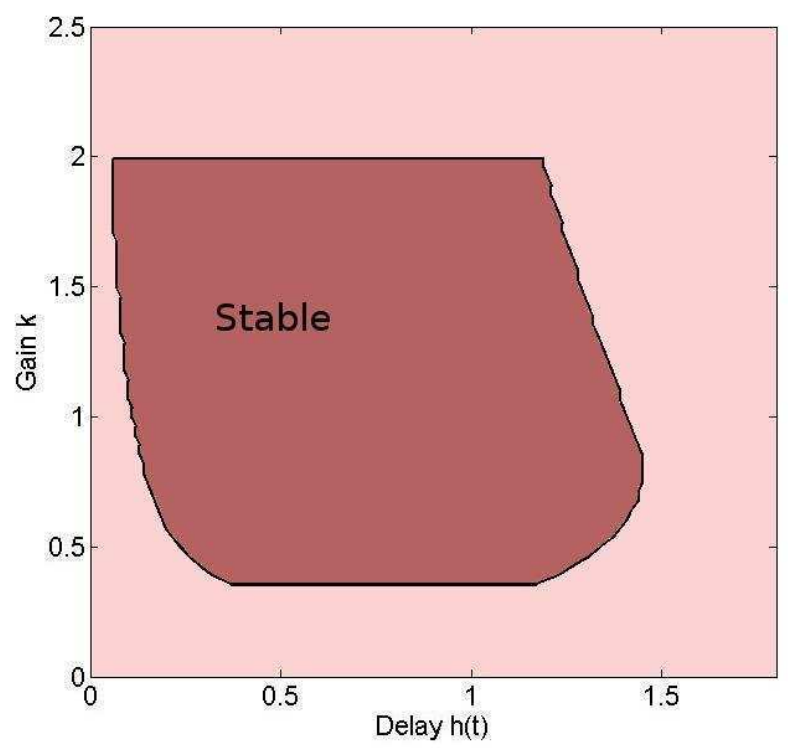

Fig. 2. Stability region of $\ddot{y}(t)-0.1 \dot{y}(t)+2 y(t)=k y(t-h(t))$ w.r.t. $k$ and $h(t)$.

\subsection{Third example: robust stability}

Consider

$$
\begin{aligned}
\dot{x}(t)= & {\left[\begin{array}{cc}
-2+\delta_{1} \cos t & 0 \\
0 & -1+\delta_{2} \sin t
\end{array}\right] x(t) } \\
& +\left[\begin{array}{cc}
-1+\gamma_{1} \cos t & 0 \\
-1 & -1+\gamma_{2} \sin t
\end{array}\right] x(t-h(t)),
\end{aligned}
$$

extracted from [32]. $\delta_{i}$ and $\gamma_{i}$ are uncertain bounded parameters:

$$
\left|\delta_{1}\right| \leq 1.6,\left|\delta_{2}\right| \leq 0.05,\left|\gamma_{1}\right| \leq 0.1,\left|\gamma_{2}\right| \leq 0.3 .
$$


Table 1. The maximal allowable delays $h_{\max }$ for system (29)

\begin{tabular}{|c||c|c|c|c|c|c|c|}
\hline $\mathrm{d}$ & 0 & 0.1 & 0.2 & 0.5 & 0.8 & 1 & $\forall d>1$ \\
\hline \hline Fridman et al (2002) [6] & 4.472 & 3.604 & 3.033 & 2.008 & 1.364 & 0.999 & 0.999 \\
\hline Wu et al (2004) [24] & 4.472 & 3.604 & 3.033 & 2.008 & 1.364 & - & - \\
\hline Kao et al (2005) [17] & 4.472 & 3.604 & 3.033 & 2.008 & 1.364 & 0.999 & - \\
\hline Fridman et al (2006) [11] & 1.632 & 1.632 & 1.632 & 1.632 & 1.632 & 1.632 & 1.632 \\
\hline Kao et al (2007) [19] & 6.117 & 4.714 & 3.807 & 2.280 & 1.608 & 1.360 & - \\
\hline He et al (2007) [28] & 4.472 & 3.605 & 3.039 & 2.043 & 1.492 & 1.345 & 1.345 \\
\hline He et al (2007) [9] & 4.472 & 3.605 & 3.039 & 2.043 & 1.492 & 1.345 & 1.345 \\
\hline Ariba et al (2009) [31] & 6.117 & 4.794 & 3.995 & 2.682 & 1.957 & 1.602 & 1.345 \\
\hline Sun et al (2010) [14] & 4.476 & 3.611 & 3.047 & 2.072 & 1.590 & 1.529 & - \\
\hline Theorem 2 & 5.120 & 4.081 & 3.448 & 2.528 & 2.152 & 1.991 & - \\
\hline
\end{tabular}

Table 3. Maximal allowable delay $h_{\max }$ for the system (31) w.r.t. $d$.

\begin{tabular}{|c||c|c|c|c|}
\hline $\mathrm{d}$ & 0 & 0.1 & 0.5 & 0.8 \\
\hline \hline$[32]$ & 0.241 & 0.234 & 0.188 & 0.110 \\
\hline$[24]$ & 1.149 & 1.106 & 0.924 & 0.760 \\
\hline$[19]$ & 1.416 & 1.302 & 0.974 & 0.829 \\
\hline Theorem 3 & 1.515 & 1.422 & 1.105 & 0.910 \\
\hline
\end{tabular}

Let us rewrite the system as in (23) with

$$
\begin{aligned}
A & =\left[\begin{array}{cc}
-2 & 0 \\
0 & -1
\end{array}\right], A_{d}=\left[\begin{array}{cc}
-1 & 0 \\
-1 & -1
\end{array}\right], \\
B=1_{2}, C & =\operatorname{diag}(1.6,0.05), C_{\mathrm{d}}=\operatorname{diag}(0.1,0.3) .
\end{aligned}
$$

Simulation results are gathered in the Table 3.

\section{CONCLUSION}

In this paper, stability analysis of a time-varying delay system was studied by means of quadratic separation. Inspired from previous work on time-delay systems with constant delay [8], novel stability criteria for time-varying delay system were provided. Using an augmented state, which emphasizes the relation between $\dot{h}$ and $(\dot{x}, \ddot{x})$, the resulting criteria were expressed in terms of a convex optimization problem with LMI constraints. Finally, numerical examples show that this method reduced conservatism and improved the maximal allowable interval on the delay.

\section{REFERENCES}

1. Y. Ariba, F. Gouaisbaut, and K. H. Johansson. Stability interval for time-varying delay systems. In the 49th IEEE Conference on Decision and Control (CDC'10), Atlanta, USA, December 2010.

2. V. B. Kolmanovskii and A. Myshkis. Introduction to the Theory and Applications of Functional Differential Equations. Kluwer Academic Publishers, 1999.

3. L.G. Bushnell. Networks and control. IEEE Control Systems Magazine, 21, February 2001.

4. R. Sipahi, S. Niculescu, C.T. Abdallah, W. Michiels, and Keqin Gu. Stability and stabilization of systems with time delay. Control Systems Magazine, IEEE, 31(1):38 -65, feb. 2011.

5. K. Gu, V. L. Kharitonov, and J. Chen. Stability of Time-Delay Systems. Birkhäuser Boston, 2003. Control engineering.

6. E. Fridman and U. Shaked. An improved stabilization method for linear time-delay systems. IEEE Trans. on Automat. Control, 47:1931-1937, November 2002.

7. A. Seuret. Lyapunov-Krasovskii functionals parameterized with polynomials. In the 6th IFAC Symposium on Robust Control Design, Haifa, Israel, June 2009.

8. F. Gouaisbaut and D. Peaucelle. Robust stability of time-delay systems with interval delays. In $46^{\text {th }}$ IEEE Conference on Decision and Control, New Orleans, USA, December 2007.

9. Y. He, Q. G. Wang, L. Xie, and C. Lin. Further improvement of free-weighting matrices technique for systems with time-varying delay. 
IEEE Trans. on Automat. Control, 52:293-299, February 2007.

10. D. Yue, Q.-L. Han, and J. Lam. Robust $H_{\infty}$ Control and Filtering of Networked Control Systems, pages 121-151. Springer London, 2008.

11. E. Fridman and U. Shaked. Input-output approach to stability and $l_{2}$-gain analysis of systems with time-varying delays. Systems \& Control Letters, 55:1041-1053, September 2006.

12. C. Briat. Robust Control and Observation of $L P V$ Time-Delay Systems. PhD thesis, INP-Grenoble, 2008.

13. H. Shao and Q.-L. Han. Less conservative delaydependent stability criteria for linear systems with interval time-varying delays. International Journal of Systems Science, pages 1-9, 2011.

14. J. Sun, G.P. G.P. Liu, J. Chen, and D. Rees. Improved delay-range-dependent stability criteria for linear systems with time-varying delays. Automatica, 46(2):466 - 470, 2010.

15. Yeong-jeu Sun. Stability criterion for a class of descriptor systems with discrete and distributed time delays. Asian Journal of Control, 33:986993, 2007.

16. Y. Ariba and F. Gouaisbaut. An augmented model for robust stability analysis of time-varying delay systems. Int. J. Control, 82:1616-1626, September 2009.

17. C. Y. Kao and A. Rantzer. Robust stability analysis of linear systems with time-varying delays. In $16^{\text {th }}$ IFAC World Congress, Prague, Czech Republic, 2005.

18. Y. Ariba, F. Gouaisbaut, and D. Peaucelle. Stability analysis of time-varying delay systems in quadratic separation framework. In The International conference on mathematical problems in engineering, aerospace and sciences (ICNPAA'08), June 2008.

19. C.-Y. Kao and A. Rantzer. Stability analysis of systems with uncertain time-varying delays. Automatica, 43(6):959 - 970, 2007.

20. D. Peaucelle, D. Arzelier, D. Henrion, and F. Gouaisbaut. Quadratic separation for feedback connection of an uncertain matrix and an implicit linear transformation. Automatica, 43(5):795804, 2007.

21. T. Iwasaki and S. Hara. Well-posedness of feedback systems: insights into exact robustness analysis and approximate computations. IEEE Trans. on Automat. Control, 43:619-630, May 1998.

22. J. Sun, G.P. Liu, J. Chen, and D. Rees. Improved delay-range-dependent stability criteria for linear systems with time-varying delays. Automatica, 46(2):466-470, 2010.

23. M.G. Safonov. Stability and Robustness of Multivariable Feedback Systems. Signal Processing, Optimization, and Control. MIT Press, 1980.

24. M. Wu, Y. He, J. H. She, and G. P. Liu. Delaydependent criteria for robust stability of timevarying delay systems. Automatica, 40:14351439, 2004.

25. J. Zhang, C. R. Knopse, and P. Tsiotras. Stability of time-delay systems: Equivalence between Lyapunov and scaled small-gain conditions. IEEE Trans. on Automat. Control, 46(3):482-486, March 2001.

26. P.-A. Bliman. Lyapunov equation for the stability of linear delay systems of retarded and neutral type. IEEE Trans. on Automat. Control, 47:327335, February 2002.

27. Y. Ebihara, D. Peaucelle, D. Arzelier, and T. Hagiwara. Robust performance analysis of linear time-invariant uncertain systems by taking higher-order time-derivatives of the states. In $44^{\text {th }}$ IEEE Conference on Decision and Control and the European Control Conference, Seville, Spain, December 2005.

28. Y. He, Q. G. Wang, C. Lin, and M. Wu. Delayrange-dependent stability for systems with timevarying delay. Automatica, 43:371-376, 2007.

29. S. Xu and J. Lam. A survey of linear matrix inequality techniques in stability analysis of delay systems. International Journal of Systems Science, 39(12):1095-1113, December 2008.

30. H. Shao. New delay-dependent stability criteria for systems with interval delay. Automatica, 45(3):744 - 749, 2009.

31. Y. Ariba and F. Gouaisbaut. Input-output framework for robust stability of time-varying delay systems. In the 48th IEEE Conference on Decision and Control, Shanghai, China, December 2009.

32. J. H. Kim. Delay and its time-derivative dependent robust stability of time-delayed linear systems with uncertainty. IEEE Trans. on Automat. Control, 46(5):789-792, May 2001. 